\title{
FIO DE PESCA: INSTRUMENTO VÁLIDO PARA PESQUISA DE NEUROPATIA DIABÉTICA PERIFÉRICA?
}

\author{
Gabriela Haana Maia dos Santos Oliveira'; Ana Mayra Andrade de Oliveira ${ }^{2}$; \\ Aline da Silva Santos ${ }^{3}$; Vitória Almeida Matos da Silva ${ }^{4}$; \\ Lília Paula de Souza Santos ${ }^{5}$ \\ 1.Bolsista PROBIC/UEFS. Graduanda em Medicina, Universidade Estadual de Feira de Santana. \\ Email: gabihaana@hotmail.com \\ 2. Professora Doutora e Orientadora do Núcleo de Pesquisa de Endocrinologia, Departamento de Saúde, \\ Universidade Estadual de Feira de Santana. Email:anamayra@uol.com.br \\ 3. Bolsista PROBIC/UEFS. Graduanda em Medicina, Universidade Estadual de Feira de Santana. \\ Email: aliness1612@gmail.com \\ 4. Bolsista PIBIC/FAPESB, Graduanda em Medicina, Universidade Estadual de Feira de Santana. \\ Email: vitoriauefs@gmail.com \\ 5. Mestre em Saúde Coletiva, Universidade Estadual de Feira de Santana. \\ Email: lipss13@hotmail.com.br
}

PALAVRAS-CHAVE:Diabetes Mellitus Tipo 2, neuropatia diabética, sensibilidade tátil.

\section{INTRODUÇÃO}

A neuropatia diabética (ND) é uma das complicações crônicas mais prevalentes do diabetes mellitus (DM). Este grupo heterogêneo de condições afetam diferentes partes do sistema nervoso periférico e autonômico (ADA, 2018). As anormalidades neurológicas ocorrem tanto no Diabetes tipo 1 quanto no tipo 2, assim como em formas de diabetes adquiridas (GALGIARDI, 2003.)

A ND não é uma entidade única e simples, mas representa um conjunto de síndromes com variadas manifestações clínicas ou subclínicas. Para fins práticos, é possível classificar a ND em somática (autonômica), focal (mononeurites e síndromes compressivas) e difusa (neuropatias proximais, polineuropatias simétricas distais, acometimento de grandes fibras e acometimento de pequenas fibras) (GAGLIARDI, 2003).

Sua prevalência é elevada e proporcional à duração da doença assim como ao seu controle, chegando a frequências de $50 \%$ de lesão neuropática em diferentes grupos de pacientes analisados nos âmbitos nacional e internacional. (SBD, 2018). Percebe-se, então, que ela compromete a sensibilidade protetora, tornando o paciente diabético mais vulnerável a traumas, visto que ele pode não perceber o estímulo agressor e, consequentemente, não se defender de tal estímulo. Esse quadro, associado às características clínicas do DM, predispõe ao surgimento de úlceras e evolução para amputação de membros (CARVALHO, 2009).

Considerando-se a relevância dessa complicação clínica, a American Diabetes Association (2018) recomenda que todos os pacientes com DM2 desde o momento do diagnóstico devem ser triados com testes clínicos simples como o do monofilamento de $10 \mathrm{~g}$ para detecção precoce de neuropatia diabética periférica (NDP), visto que a detecção e identificação precoce do processo neuropático oferecem uma oportunidade crucial para o paciente diabético, no sentido de ativamente procurar o controle glicêmico ótimo e implementar cuidados corporais com redução da morbidade (GAGLIARDI, 2003). Tais orientações fundamentam-se no fato de que o rígido 
controle glicêmico é a única alternativa reconhecida para retardar a progressão da ND no DM2 (ADA, 2018).

A profilaxia das complicações neuropáticas deve ser iniciada pela identificação do grau de neuropatia e, portanto, do déficit neurológico. Isto é possível, atualmente, por meio de avanços na avaliação de sensibilidade cutânea nos membros inferiores (CARVALHO, 2009). Um exemplo é o uso já consolidado do monofilamento de SemmesWeinstein (estesiômetro) que oferece medidas quantitativas como resultado (CARVALHO, 2006).

Diante do exposto, inquieta-nos saber se o fio de pesca (um monofilamento, doméstico) poderia ser viável na avaliação da sensibilidade protetora e conhecer as possíveis vantagens deste, já que o custo do monofilamento classicamente usado, muitas vezes inviabiliza sua utilização mais abrangente.

\section{MATERIAL E MÉTODOS OU METODOLOGIA (ou equivalente)}

Trata-se de um estudo prospectivo de análise da aplicabilidade do fio de pesca de $4 \mathrm{~cm}$ para detecção de perda da sensibilidade protetora em portadores de ND a ser realizado no Centro de atendimento ao diabético hipertenso (CADH). Foram incluídos pacientes com diagnóstico de DM2 há mais de 10 anos, que não tivessem realizado cirurgia vascular prévia, presença de condições cognitivas adequadas para responderem às perguntas clínicas do trabalho, ou que não apresentassem dúvidas quanto à etiologia da ND.

Inicialmente realizou-se o levantamento de fio de pesca disponível no mercado nacional que apresentasse características similares quanto à composição e características de flambagem do monofilamento de $10 \mathrm{~g}$. Esse estudo foi realizado em laboratório com balança digital de precisão (marca OHAUS modelo Max com capacidade de $210 \mathrm{~g}$ e $\mathrm{d}=1 \mathrm{mg}$ ). Encontrou-se flambagem, confirmada, de10 g no fio fabricado com Nylon 6 (Homopolímero) com diâmetro de $0,50 \mathrm{~mm}$ destinado à fabricação de redes de pesca e pesca esportiva, com vara (marca TREVO ${ }^{\circledR}$, Equipesca, SP- Brasil), que é fornecido em bobinas com 250 g. nomeou-se este de fio "monofilamento doméstico" (MFD).

Foi realizado o screening de pé de risco para pé diabético feito com o monofilamento disponível comercialmente (MFC) que utilizamos na rotina do serviço, e, na mesma população se aplicou o fio "doméstico" (MFD), observando se existia concordância ou não das respostas referentes à percepção do estímulo realizado, respostas: sim/não.

O monofilamento foi aplicado perpendicularmente à superfície da pele com força suficiente para encurvá-lo na mão, cotovelo, ou fronte do paciente para que o paciente entendesse de que forma seria realizado o teste nos seus pés. O filamento foi então aplicado nas projeções das porções ventrais do hálux, terceiro dedo e projeção das cabeças dos $1^{\circ} \mathrm{e} 3^{\circ} 5^{\circ}$ metatarsianos, não excedendo o tempo de 2 segundos. A aplicação foi repetida 2 vezes no mesmo local, alternando com pelo menos, 1 aplicação simulada, fazendo-se 3 perguntas por local de aplicação. A sensibilidade era considerada positiva quando o paciente respondia corretamente todas as aplicações e, ausente, se pelo menos 1 das 3 perguntas fossem respondidas de maneira incorreta.

Os cálculos foram realizados com o auxílio do software SPSS (StatisticalPackage for Social Sciences), versão 22. As variáveis contínuas foram 
expressas em valor médio \pm desvio padrão (DP) e as categóricas como frequências ou proporções. Utilizando o teste qui quadrado de person e o teste de Kappa para avaliar a concordância.

De acordo com os aspectos éticos o estudo foi conduzido em conformidade com o Comitê de Ética e Pesquisa (CEP) e todos os pacientes assinaram o Termo de Consentimento Livre e Esclarecido (TCLE). Os pacientes possuíram autonomia sobre a participação de qualquer etapa da pesquisa. As informações obtidas foram analisadas em conjunto com as de outros pacientes, e não será divulgada a identificação de nenhum paciente. O estudo ao qual o projeto se vincula foi aprovado pelo CONSEPE local (049/2013).

\section{RESULTADOS E/OU DISCUSSÃO}

O estudo contou com a participação de 116 indivíduos com idade média de 65,8 \pm 9 anos, IMC médio de $28,4 \pm 5,6 \mathrm{~kg} / \mathrm{m} 2$, sendo $75 \%$ da amostra composta por mulheres. Com relação à etnia, declararam-se brancos, pretos, pardos, amarelos e indígenas $8,6 \%, 37,9 \%, 50,9 \%, 1,7 \%$ e $0,9 \%$ respectivamente. A média do tempo de diagnóstico de DM2 foi de 18,7 $\pm 6,6$ anos. Tabagistas representava 26,7\% das pessoas entrevistadas, $87 \%$ eram hipertensos e $28,1 \%$ apresentaram problemas cardiovasculares.

O screening de pé de risco realizado, com o MFC teve 53,4\% de resultados positivos e o MFD 56\% (Tabela 1). A concordância entre os dois instrumentos foi de $51,7 \%$, Kappa $=0,739$ (Tabela 2).

\begin{tabular}{lcc}
\multicolumn{3}{l}{ Tabela 1: Concordância entre o teste com o MFC* e M } \\
\hline Variáveis & n & $\%$ \\
\hline Monofilamento & & \\
$\quad$ Negativo & 54 & 46,6 \\
Positivo & 62 & 53,4 \\
Fio de pesca & & \\
$\quad$ Negativo & 51 & 44,0 \\
Positivo & 65 & 56,0 \\
\hline
\end{tabular}

*MFC: Monofilamento Comercial** MFD: Monofilamento Doméstico

Tabela 2: Validação entre teste de MFC* e MFD**.

\begin{tabular}{lcc}
\hline Validação & $\mathbf{n}$ & $\mathbf{\%}$ \\
\hline Concordante & 60 & 51,7 \\
Não concordante & 56 & 48,3 \\
\hline
\end{tabular}

*MFC: Monofilamento Comercial** MFD: Monofilamento Doméstico

\section{CONSIDERAÇÕES FINAIS}

Resultados semelhantes foram obtidos quando comparados os testes com MFC e MFD, que mostram o MFD como uma alternativa real à MFC e, provavelmente, podem ser consolidados como uma nova ferramenta no diagnóstico de pé em risco na população diabética. 


\section{REFERÊNCIAS}

American Diabetes Association - ADA. DiabeticNeuropathy- Diabetes Care 2017; v. 40, pag 136-154.

GAGLIARDI, A. R. T. Neuropatia diabética periférica. J Vasc BR, São Paulo, v. 2, n. 1, p. 6774. 2003.

Sociedade Brasileira de Diabetes. Diretrizes da Sociedade Brasileira de Diabetes. Grupo Editorial Nacional, 2016.

CARVALHO, V. F. et. al. Limiar de sensibilidade cutânea dos pés em pacientes diabéticos através do pressurespecifiedsensorydevice: uma avaliação da neuropatia. Rev. Assoc. Med. Bras., São Paulo, v. 55, n. 1, p. 29-34, 2009.

American Diabetes Association - ADA. Standards of medical care in diabetes-2016. Diabetes Care2017.

CARVALHO, G. A.; CHIERICHETTI, H.S. Avaliação da sensibilidade cutânea palmar nas aplicações de crioterapia por bolsa de gelo e bolsa de gel. Rev. Bras. Ciên. Mov., p. 23-32, 2006.

SILVA, C. A. M.; PEREIRA, D. S.; ALMEIDA, D. S. C.; VENÂNCIO, M. I. L. Pé diabético e avaliação do risco de ulceração. Rev. Enf. Ref., Coimbra, v. 4, n. 1, 2014. 